\title{
Composite material Hollow Antiresonant Fibers
}

\author{
Walter Belardi ${ }^{*}$, Pier Sazio, Francesco De Lucia and Francesco Poletti \\ Optoelectronics Research Centre, University of Southampton, Highfield, Southampton, Hampshire. SO17 1BJ, UK \\ *w.belardi@soton.ac.uk
}

\begin{abstract}
We analyze air-core fiber designs with different materials in the cladding, including structures with an extended bandwidth and hybrid semiconductor/glass fibers with very low attenuation. We report on the first realization of this fiber type.

OCIS codes: (060.2280) Fiber design and fabrication; (060.2400) Fiber properties; (060.4005) Microstructured fibers.
\end{abstract}

\section{Introduction}

Recently the use of fiber structures comprising a limited number of glass tubes within an outer glass jacket has led to the realization of fibers with low attenuation in the visible[1], near [2] and mid-infrared spectral ranges[3], and ultralarge transmission bandwidth [1, 2, 3]. In this work we seek to explore a novel form of this fiber type, by using multiple materials for the fiber cladding. We numerically study the transmission properties of these Composite Material Anti-Resonant Fibers (CM-ARFs), by introducing the notion of "single equivalent membrane". We apply this concept to the design of novel forms of antiresonant fibers, which includes air/silica fiber structures with an extended transmission bandwidth as well as hybrid semiconductor/glass based ARFs. Similarly to previous works on semiconductor optical fibers $[4,5]$, we seek to realize functional optical fibers, e.g. an optical phase modulator, by modifying the fiber guidance properties (its effective refractive index) through the insertion of a semiconductor in the fiber cladding area. We numerically demonstrate that, differently from previous structures with silicon in the cladding area [5], this novel type of silicon based ARF can have very low attenuation $(<0.1 \mathrm{~dB} / \mathrm{m})$. We also report on the first fabrication of a silicon/borosilicate based ARF, proving the feasibility of our target.

\section{Multiple membranes antiresonant fibers}

The inset in Fig. 1(A) shows a silica based ARF studied in [2] with an additional internal membrane (in red) with thickness $t$ and refractive index $n_{2}=2$. The dependence of its leakage loss on the thickness $t$ in Fig.1(A), at a wavelength of $2.7 \mu \mathrm{m}$, proves the anti-resonant behavior of this CM-ARF. Fig. 1(B) shows the evolutions of the leakage loss when additional antiresonant glass membranes $(2,3$ or 5$)$ are added to the basic structure [2]. Fiber designs where the thickness of the initial single silica membrane is simply increased 2, 3 or 5 times (red, black and blue) are shown to have an almost identical behavior to those ones with respectively 2,3 or 5 alternating $\left(n_{1} / n_{2}\right)$ antiresonant glass membranes (cyan, green and purple). While the minimum loss is almost unchanged, the bandwidth of the CM-ARFs is inversely dependent on the thickness of their equivalent single glass membrane.
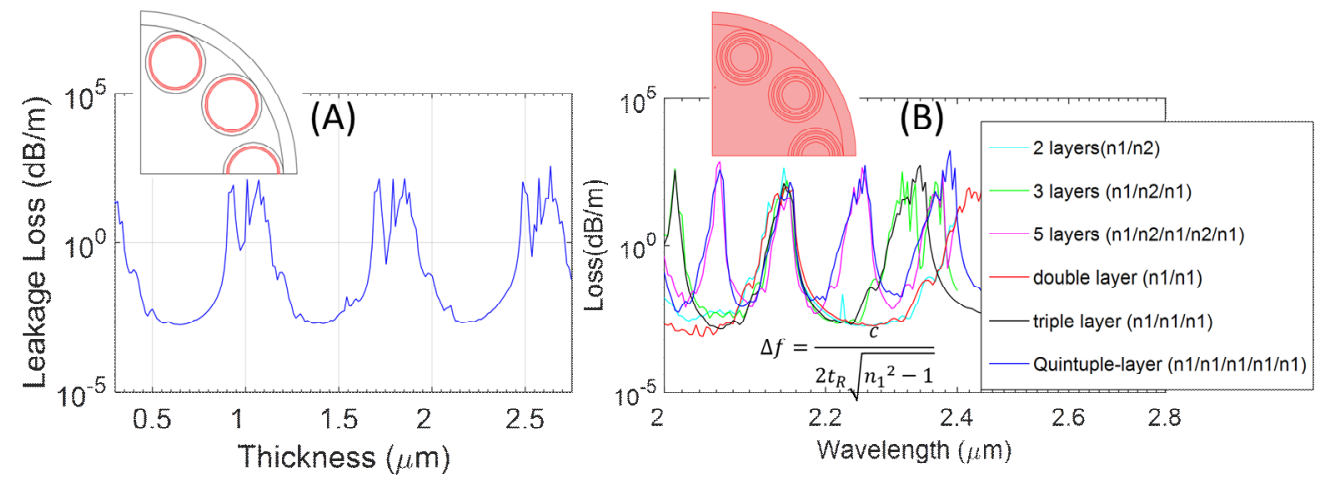

Figure 1. Composite Material hollow optical fibers with antiresonant guidance properties.

\section{Equivalent membrane}

The blue plot in Fig. 2(A) shows the leakage loss of a CM-ARF made of 2 glass membranes with different refractive index $\left(n_{1}=1.414, n_{2}=2\right)$ and different thickness $\left(t_{l}=0.66 \mathrm{~mm}, t_{2}=0.99 \mathrm{~mm}\right)$, while the red plot shows the loss of an $\mathrm{ARF}$ with a single equivalent glass membrane whose thickness $i s t_{e}=t_{1}+t_{2}$ and whose equivalent refractive index $n_{e}$ is calculated by using the formula in the inset of Fig. 2, where $S_{1}$ is the total glass surface of the first material and $S_{2}$ is the total glass surface of the second material. The overlap of the blue and red curve demonstrates the validity of this equivalence. By extrapolating this rule to the case of having air $(n=1)$ as the second material, it is possible to 
obtain an equivalent glass refractive index which is less than that of silica glass, thus allowing for the extension of the transmission bandwidth of hollow core ARFs [6]. Such air/silica membrane design, shown in Fig. 2(B), has a number of air holes of radius $r$ within the silica glass membrane (of thickness $t$ ). The plots in Fig. 2(B) show how increasing the hole radius $r$ one can decrease the equivalent glass refractive index and thus enlarge the transmission bandwidth (as in [6], the red, green and black plots were also shifted in wavelength, due to the change of $n_{e}$ ).

(A)
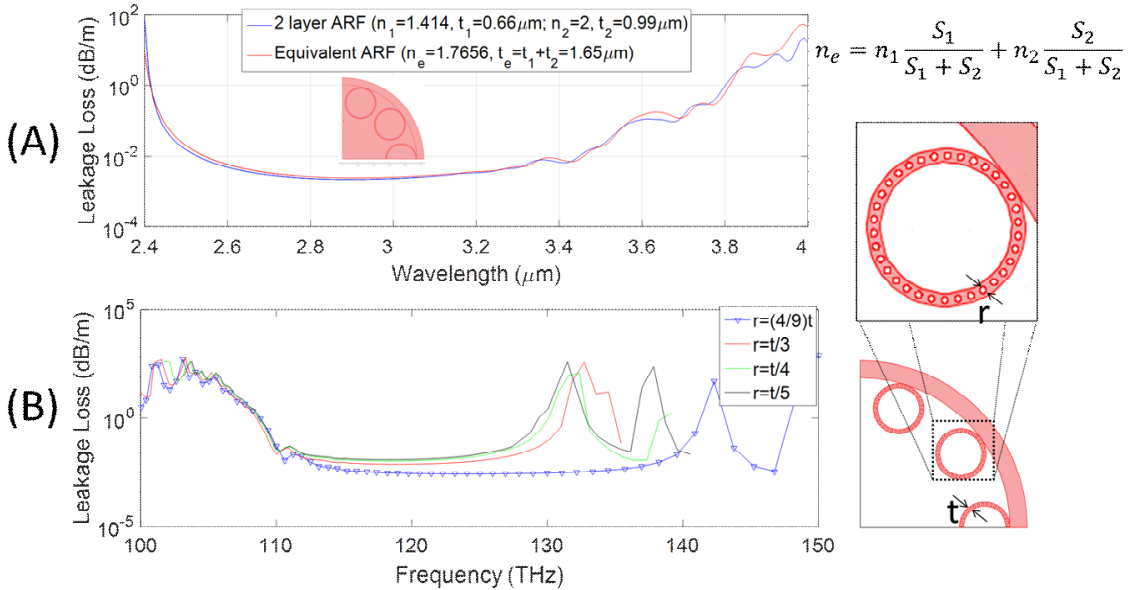

Figure 2. (A) A CM-ARF is equivalent to an ARF with a core boundary of equivalent thickness and refractive index. (B) Design of an ARF with an air/silica core boundary. Since its equivalent refractive index is less than the refractive index of silica glass, this fiber has an extended transmission bandwidth.

\section{Silicon based hollow antiresonant fiber}

One possible application of CM-ARFs is the realization of hollow core fibers comprising an internal and external silicon ( $\mathrm{Si}$ ) membrane $\left(\mathrm{n}_{2}=3.4, \mathrm{t}_{2}=0.2 \mu \mathrm{m}\right)$, as sketched in the inset of Fig. 3 (the red lines represent Si membranes and the white space between them is the original silica membrane [2]). The plots show that the calculated minimum attenuation of these novel hybrid fibers is below $0.1 \mathrm{~dB} / \mathrm{m}$ (leakage loss is dominating when assuming a Si attenuation of $100 \mathrm{~dB} / \mathrm{cm}$ ), almost 3 orders of magnitude lower than that of previous hybrid silicon/glass optical fibers $[4,5]$, potentially opening interesting opportunities in the field of functional fiber devices. The optical images on the right hand side of Fig. 3 show the transversal section of the first Si based ARFs: white lines represent silicon deposited by High Pressure CVD [4] along few $\mathrm{cm}$ of borosilicate based ARFs, proving the feasibility of our target.

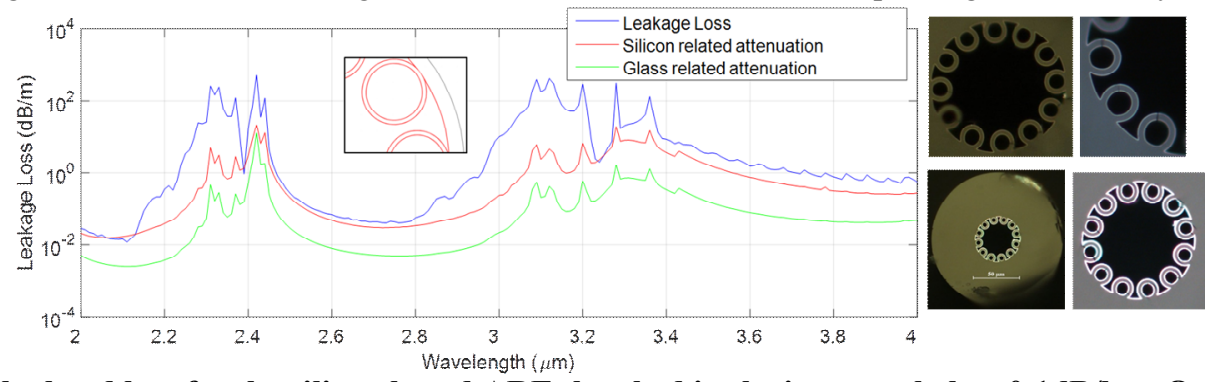

Figure 3. Calculated loss for the silicon based ARF sketched in the inset are below $0.1 \mathrm{~dB} / \mathrm{km}$. On the right hand side are some optical images of the first silicon based ARFs.

\section{Conclusion}

We have discussed some designs of CM-ARFs comprising an optical core boundary with an equivalent refractive index lower or higher than that of silica glass. This has shown the potential of realizing hollow core fibers with an extend transmission bandwidth or with the additional functionalities offered by semiconductors.

\section{References}

[1] W. Belardi, Journal of Lightwave Technology, vol. 33, no. 21, pp. 4497-4503 (2015)

[2] J. Hayes et al. Optical Fiber Communication Conference, Anaheim, postdeadline paper Th5A.3 (2016)

[3] W. Belardi et al., Optics Express vol. 22, no. 8, pp. 10091-10096 (2014)

[4] P. Sazio et al., Science, Vol.311, no 5767, pp.1583-1586, (2006).

[5] N. Healy et al, Optics Express, Vol. 19, no 11, pp. 10979-10985 (2011)

[6] A. Argyros et al., Optics Express 16, no 8, pp. 5642-5648 (2008) 